\title{
Tendências da investigação epidemiológica em doenças crônicas
}

\author{
Epidemiological research trends \\ in chronic diseases
}

\footnotetext{
1 Departamento de Epidemiologia, Instituto de Medicina Social, Universidade do Estado do Rio de Janeiro. Rua São Francisco Xavier 524, $7 \underline{0}$ andar, Rio de Janeiro, $R J$ 20559-900, Brasil. gulnar@uerj.br
}

\begin{abstract}
Identification of adult risk factors has contributed to a decrease in the occurrence of cardiovascular diseases and some types of cancer, particularly in developed countries. However, the prevailing etiologic model emphasizes risk factors associated with life style and occupational exposures. This emphasis leads to an excessive individualization of risk, weakening the link between epidemiology and public health. This article comments on the opinions of epidemiologists concerning the failures, limitations, and evolution of modern epidemiology that have helped redirect the field's theoretical underpinnings. Alternatives to the current model tend to integrate findings from molecular biology with classic risk factors and early adverse conditions in life, taking into account all these causal influences in hierarchical levels of organization. The author identifies and comments on three important approaches in this evolution: molecular epidemiology, the theory of early life factors, and multilevel models.
\end{abstract}

Key words Chronic Disease; Molecular Epidemiology; Risk Factors

Resumo A identificação de vários fatores de risco da vida adulta contribuiu, sobretudo nos países desenvolvidos, para a diminuição da ocorrência de doenças cardiovasculares e de alguns tipos de câncer. O modelo etiológico mais adotado enfatiza no entanto, os fatores de risco relacionados ao estilo de vida e às exposições ocupacionais. Esta ênfase vem levando a uma excessiva individualização do risco, com um conseqüente enfraquecimento do vínculo da epidemiologia com a saúde pública. São comentadas neste artigo as opiniões de epidemiologistas que fazem uma reflexão sobre as falhas, os limites e os desdobramentos da epidemiologia moderna que têm contribuído para uma reorientação teórica da disciplina. Identifica-se assim, que as alternativas ao modelo atual devem buscar uma integração entre os achados de biologia molecular com os fatores de risco classicamente concebidos e com condições adversas em etapas precoces da vida, considerando todas estas influências causais em níveis hierarquizados de organização. São destacadas e comentadas três importantes vertentes neste processo de evolução: a epidemiologia molecular, a teoria dos eventos precoces na vida e os modelos multinível.

Palavras-chave Doença Crônica; Epidemiologia Molecular; Fatores de Risco 


\section{Introdução}

A epidemiologia foi fundamental no controle das doenças infecciosas e na descoberta de muitos fatores associados a vários tipos de câncer, à doenças cardiovasculares e a outras doenças crônicas. Até a primeira metade do século $\mathrm{XX}$, os fatores mais importantes relacionados às doenças infecciosas como a cólera, a malária e mesmo a algumas crônicas (escorbuto e pelagra) eram de natureza macro-ambiental e a prevenção dependia de medidas coletivas de saúde pública, que englobavam atuação de outros profissionais não-médicos como engenheiros, químicos, entomologistas, planejadores (Adami \& Trichopoulos, 1999). O grande desenvolvimento metodológico da epidemiologia, após a segunda guerra mundial, possibilitou a identificação de fatores envolvidos na etiologia de muitas enfermidades crônicas e a ênfase da epidemiologia se voltou para o micro-ambiente onde prevaleceu a investigação de fatores de risco da vida adulta. O estudos de Doll \& Hill, Wynder \& Graham, os quais tiveram suas primeiras publicações no início da década de 50, foram importantes para o declínio do tabagismo e das doenças associadas ao fumo (Peto, 1999). A partir daí, a epidemiologia na tentativa de explicar a etiologia das doenças crônicas, vem se concentrando na medida de risco de um largo espectro de fatores associados ao estilo de vida e a exposições ocupacionais.

O enfoque centrado no indivíduo tem favorecido uma aproximação maior da epidemiologia à clínica, com um conseqüente afastamento da saúde pública, passagem que não tem acontecido silenciosamente. Os questionamentos sobre os limites e o futuro da epidemiologia moderna são cada vez mais freqüentes entre epidemiologistas. Há os que consideram que esta discussão reflete em parte, a crise de paradigma por que passa a epidemiologia (Barreto, 1998; Schwartz et al., 1999; Susser \& Susser, 1996b). As possibilidades de novos conhecimentos sobre fatores de risco com associação forte e alta especificidade não vêm acontecendo (Barreto, 1998) e, mesmo que existam conhecimentos disponíveis, a avaliação dos resultados de intervenções tem se mostrado em geral, insuficiente (Susser, 1995)

De certa forma, o que se percebe dentro do debate sobre os rumos da epidemiologia, presente nos periódicos da área, é de que não se trata de uma crise aguda nem irremediável, mas sim de um processo decorrente da própria evolução da disciplina e da saúde pública, onde os argumentos se baseiam na percepção de que existe uma inquietação na tentativa de en- contrar caminhos que venham a contribuir de forma mais efetiva na compreensão sobre o processo saúde/doença.

\section{Evolução da investigação epidemiológica}

Susser \& Susser (1996a, 1996b) analisam a evolução da epidemiologia moderna através de três eras, considerando que cada uma delas teria um paradigma dominante característico que seria responsável pela definição da abordagem preventiva de cada era. Assim, a primeira foi representada pelas estatísticas sanitárias que teve como paradigma o miasma. Na segunda estaria a epidemiologia das doenças infecciosas trazendo a teoria do germe como paradigma dominante. A terceira era, a atual, é caracterizada pela epidemiologia das doenças crônicas que teria como paradigma a caixa preta, que simboliza o paradigma da epidemiologia dos fatores de risco para as doenças crônicas. Para os autores, nas duas primeiras eras a saúde pública teria papel central, mas na era presente a ênfase dada à saúde pública estaria mais tênue. Prevêem ainda os autores um futuro fechamento desta terceira era, que daria lugar a uma nova etapa marcada pela eco-epidemiologia, onde o desdobramento em um paradigma diferente se tornará essencial. Este paradigma estaria se formando dentro dos limites de muitos níveis de organização (molecular, social e individual) e por isso seria denominado de "caixas chinesas" que integrariam mais de um simples nível no desenho, análise e interpretação. Este paradigma poderia sustentar e refinar a orientação da epidemiologia para a saúde pública.

Dois anos mais tarde, Susser (1998) volta a se referir ao atual paradigma da caixa preta, considerando que sua evolução estaria sendo menos proveitosa e gradativamente substituída pelo crescimento das epidemiologias molecular e global baseado nos sistemas de informação. Esta dicotomia poderia dividir a epidemiologia em duas distintas disciplinas, ao mesmo tempo em que o interesse pela saúde pública estaria se enfraquecendo. Para Susser, a eco-epidemiologia teria o potencial de juntar estas duas vertentes.

Alguns autores são pessimistas em relação ao papel que a epidmiologia vem exercendo. Shy (1997) considera que a epidemiologia acadêmica falhou em desenvolver os métodos científicos e a base de conhecimento para prevenir doenças e promover a saúde através de esforços organizados da comunidade, o que se- 
ria sua missão fundamental dentro da saúde pública; a epidemiologia acadêmica estaria cometendo a falácia biomédica, que seria inferir que a doença nas populações pode ser entendida pelo estudo dos fatores de risco em indivíduos. Esta abordagem centrada no indivíduo não tem respondido muitas questões que não são determinadas apenas no nível individual, mas que têm uma grande interdependência com fatores sociais, culturais, econômicos e ambientais. Em termos preventivos o enfoque individualizado leva à culpabilização da vítima e pode produzir intervenções nocivas (Pearce, 1996).

Em contraposição ao suposto argumento de estagnação da epidemiologia dos fatores de risco, Trichopoulos (1996) mostra exemplos de recentes avanços na epidemiologia cardiovascular como a elucidação do papel dos lipídios sangüíneos, a documentação dos efeitos da aspirina, do etanol, da homocisteína e outros. $\mathrm{Na}$ área do câncer, foi também recente a caracterização dos vírus $\mathrm{B}$ e $\mathrm{C}$ da hepatite e de certas cepas de papilomavírus como definitivos carcinógenos. Outro exemplo seria o da proteção dada por consumo de frutas e vegetais para vários tipos de câncer, que só foi totalmente aceita nos últimos vinte anos. Encara o autor portanto, o futuro da epidemiologia de uma forma mais otimista com argumentos favoráveis que se anunciam: 1) aumento na ênfase da especificidade através de grandes estudos que permitirão a distinção entre resultados falsos e verdadeiros; 2) a epidemioloiga clínica deve melhorar a forma como a medicina e os serviços de saúde são definidos, implementados e avaliados; 3 ) a descoberta de genes que predispõem a doenças exigirá a urgente identificação de fatores exógenos que interajam com estes genes, levando ao aparecimento de doenças na espécie humana; e 4) conceitos que se aprimoram, como por exemplo ênfase nos eventos precoces de vida, podem enriquecer o futuro da pesquisa epidemiológica e os desdobramentos teóricos podem contribuir na elucidação de grandes enigmas, como as diferenças inexplicáveis entre morbidade e mortalidade entre grupos sócio-econômicos.

É inegável que o advento de novas técnicas e a crescente identificação de genes, aumentou de forma exponencial a pesquisa sobre a genética das doenças e de seus mecanismos moleculares. Segundo Diez-Roux (1998a) a epidemiologia hoje pode estar de fato, à beira de um novo paradigma, o genético. Os genes recentemente descobertos são tidos como importantes fatores de risco para doenças, mas também de comportamento como atividade física (Pe- russe et al., 1989) e características psicossociais (Kendler, 1997). No entanto, ao mesmo tempo parece que vem ressurgindo o interesse sobre a origem social das doenças, a se medir pelo aumento de publicações que avaliam as diferenças na saúde relacionadas às classes sociais. As explicações sociais têm competido com outras que enfatizam os comportamentos pessoais e biológicos e, talvez hoje o contraste entre estes níveis de explicação tenha se tornado mais evidente do que nunca (Diez-Roux, 1998a).

\section{Tendências atuais}

Dentro da progressiva individualização do risco, as influências ambientais e sociais são pouco consideradas ou ficam geralmente, em segundo plano. O estilo de vida e o comportamento são encarados como escolha individual dissociados do contexto social. O processo de causalidade das doenças não é uma propriedade de agentes, mas de sistemas complexos ocorrendo dentro do contexto populacional. A constatação de que os fatores de risco clássicos só explicam parcialmente as desigualdades sociais e geográficas da distribuição das doenças crônicas, tem levado a um crescente investimento em marcadores genéticos, em outros fatores de ordem psicossocial e ambiental da vida adulta, na análise do consumo alimentar e de diversos fatores pré-natais e da infância. Nesse sentido é aqui pertinente destacar três marcantes vertentes que se colocam como importantes tendências na evolução da pesquisa epidemiológica sobre causalidade de doenças crônicas: a epidemiologia molecular, a teoria dos eventos precoces na vida e os modelos multinível.

\section{Epidemiologia molecular}

No ponto de vista dos epidemiologistas, "epidemiologia bioquímica ou molecular” é a incorporação de biomarcadores na pesquisa epidemiológica analítica (Hulka et al., 1990). Após a revolução da biologia molecular foi possível identificar biomarcadores em níveis moleculares, e esta precisão permite o desenvolvimento de modelos de compreensão da causalidade das doenças. O avanço da biologia molecular aumentou muito o conhecimento sobre as doenças genéticas, incluindo o mapeamento de genes responsáveis pela herança mendeliana, como por exemplo os da fibrose cística. Desenvolveu ainda, modelos de predição de doenças como os mecanismos de múltiplos estágios da 
carcinogênese, tornando claro que as neoplasias malignas geralmente aparecem por causa de acumulações em série de danos a genes em uma única célula (Ellsworth et al., 1997).

A velocidade crescente de produção de conhecimento sobre a etiologia das doenças em biologia molecular, requer dos epidemiologistas um enorme esforço para compreender as possibilidades e limitações dos métodos e técnicas (Adami \& Trichopoulos, 1999). Segundo Susser (1999) a biologia molecular hoje por ser considerada inquestionável, definitiva e específica traz a mesma ilusão da teoria do germe: a capacidade de explicar tudo. Na verdade as explicações hoje disponíveis são ainda limitadas para comprovar a causalidade de grande parte das doenças crônicas. A viabilidade e aplicabilidade de estudos epidemiológicos incorporando dados de biologia molecular, ainda são questionáveis porque dependem de técnicas que muitas vezes não permitem a inclusão de um número grande de indivíduos, o que torna complicado inferir resultados a partir de dados observados (Hulka et al., 1990). Além do que, os achados em epidemiologia se tornam mais consistentes quando se repetem em outros estudos e conduzidos em grupos diferentes de população.

Para os que defendem a idéia de que o futuro progresso da pesquisa epidemiológica se daria por conta da elucidação dos mecanismos biológicos, a soma de conhecimentos poria fim à epidemiologia da caixa preta (Savitz, 1994). Ao se encampar a biologia como disciplina preponderante na comprovação das relações causais em epidemiologia, pode levar ao erro de reduzir o espectro de compreensão mais global do processo causal. A evidência biológica como fonte de hipóteses epidemiológicas pode enfraquecer as hipóteses geradas pelos dados descritivos, pela intuição ou observação clínica (Savitz, 1994). Os estudos epidemiológicos são certamente, viáveis mesmo que os mecanismos não sejam compreendidos em parte ou em sua totalidade. Certas situações de risco ou proteção são muitas vezes difíceis de serem simuladas em laboratório, embora possam ter grande importância para a saúde pública.

\section{Teoria dos eventos precoces na vida}

Embora existindo a hipótese de que a riqueza material aumentaria o risco de doenças cardiovasculares, foi observado na Inglaterra que estas doenças eram mais comuns entre indivíduos mais pobres. Partindo deste paradoxo várias pesquisas passaram a relacionar a priva- ção na infância, e não a riqueza, com o aparecimento de doenças crônicas. Foram analisadas assim, condições ambientais e experiências durante a adolescência, infância, primeiro ano de vida e pré-natal associadas à pobreza que poderiam tornar indivíduos mais suscetíveis a estas doenças, independente ou em combinação com fatores da vida adulta (Kuh \& Ben-Sholomo, 1997). Diversos estudos mostraram associações entre fatores pré e perinatais e risco de doenças cardiovasculares, bronquite crônica, disfunções tiroidianas, alergias, câncer de estômago, suicídio e aparecimento de fatores de risco na vida adulta (aumento de pressão sangüínea, intolerância à glicose, hipercolesterolemia, obesidade, etc.).

Com base em séries de coortes históricas e extrapolando resultados de estudos em animais, Barker (1990) propôs que a doença crônica seria biologicamente programada no útero ou no início do primeiro ano de vida e denominou "programming" o processo em que o estímulo ou a agressão, em períodos críticos de desenvolvimento, teria repercussão na estrutura ou na função de órgãos, tecidos e sistemas orgânicos. No caso da doença coronariana por exemplo, a hipótese sugerida é de que a subnutrição fetal na metade ou no final da gestação levaria ao retardo do crescimento intra útero, aumentando o risco de doença futura pela programação de pressão arterial, do metabolismo de colesterol e da coagulação sangüínea ou por um processo hormonal (Barker, 1995).

Muitos estudos fortaleceram a hipótese do programming, na qual geralmente o baixo peso ao nascer se associa ao risco aumentado de doença cardiovascular, e o sobrepeso, ao risco de câncer de mama e outros cânceres. Estes estudos receberam porém, muitas críticas. Joseph \& Kramer (1996) foram contundentes ao afirmar que várias apreciações diretas ou indiretas desta evidência, sugeriam que as associações encontradas eram mais devidas a viéses do que a uma relação causal. Entre as falhas, apontaram que os estudos eram acometidos por viés de seleção, não controlavam de forma adequada o confundimento gerado pelas conseqüências da privação social na saúde, além de terem inconsistências nas hipóteses testadas e nos métodos de análise.

Robson (1992) no entanto, defende que o grupo de trabalho que sustenta a hipótese de programming avançou rapidamente partindo dos estudos ecológicos para os individuais, oferecendo evidência para a hipótese em relação a importantes variáveis fisiológicas e bioquímicas, o que pode estabelecer a existência de relações específicas entre a experiência pre- 
coce e doenças ou modificações fisiológicas tardias. O desenvolvimento de estudos individuais e a incorporação de resultados de estudos experimentais para a avaliação desta hipótese, foi importante por que permitiu o controle de fatores de confundimento, o que não era feito nos estudos iniciais. Isto pôde ser observado tanto nos estudos ecológicos, onde por exemplo o acidente vascular cerebral se correlaciona com mortalidade materna e neonatal, a bronquite crônica com mortalidade pós-neonatal e a doença isquêmica do coração com mortalidade neonatal e pós-neonatal; como nos estudos individuais, quando se observou que o crescimento fetal ou neonatal está relacionado à hipertensão arterial, intolerância à glicose e ao aumento de colesterol e fibrinogênio (Barker et al., 1989, 1990, 1992).

Kuh \& Ben-Shlomo (1997) oferecem uma proposta que seria complementar à hipótese de Barker e colaboradores, considerando que são importantes não apenas os estímulos ou agressões ocorridos na vida intra-uterina ou no primeiro ano de vida, mas sim a acumulação de riscos durante o curso da vida. Esta concepção de risco seria diferente do programming por que não requer a existência de período crítico, ou seja, alguma condição desfavorável que tenha ocorrido em momentos específicos na vida intra-uterina ou na infância precoce. Mais ênfase é dada a uma variedade de experiências biológicas e sociais na infância, adolescência e na vida adulta precoce, do que no estilo de vida e nos modelos programming. Como exemplo mostram que o baixo peso ao nascer está associado à alta pressão sangüínea e à resistência à insulina na vida adulta, mas recentemente, foi sugerido que esta relação é mais forte entre homens e mulheres que têm sobrepeso. Isto levanta a hipótese de que fatores relacionados ao sobrepeso ao longo da vida podem potencializar o risco.

Estudo recente fortalece a teoria do programming com base em dados de larga escala, oferecendo evidência consistente para a hipótese de relação entre eventos precoces no período gestacional e doença isquêmica do coração (Leon et al., 1998). Em coorte de 15.000 homens e mulheres nascidos entre 1915-29, acompanhados até 1995, a mortalidade por doenças cardiovasculares se associou ao tamanho ao nascer por idade gestacional em homens, mesmo após controle por confundimento sócioeconômico. Nesta mesma linha, no Brasil, Barros \& Victora (1999) encontraram após seguimento de quinze anos da coorte dos 6.000 recém-nascidos em Pelotas, relação inversa, estatisticamente significativa, entre peso ao nas- cer por idade gestacional e pressão sangüínea após controle por índice de massa corporal e altura.

\section{Modelos multinível}

O desenvolvimento de modelos multinível tem viabilizado a análise de estudos que integrem indivíduos, dentro de seus grupos ou contextos sociais, examinando os efeitos combinados tanto das variáveis individuais como das de grupos. Esta nova abordagem não tem o objetivo de substituir, mas de complementar e enriquecer o enfoque hoje dominante, indo além das explicações individuais e biológicas (DiezRoux, 1998b). Nos modelos multinível as variáveis se estruturam na população de forma hierárquica e os dados amostrais são tratados como exemplos de múltiplos estágios dentro desta estrutura hierarquizada (Hox, 1995).

As variáveis de exposição utilizadas podem ser definidas em qualquer nível da hierarquia sendo que algumas podem ser medidas diretamente no seu estágio natural, podendo passar de um para outro por agregação ou desagregação. Por exemplo, o indicador sócio-econômico pode ser medido individualmente (escolaridade, renda per capita) e aí seria considerado uma variável de primeiro nível, medida diretamente no indivíduo e portanto desagregada. Pode-se porém, definir que este indicador será medido no estágio intermediário e aí seriam escolhidas variáveis de segundo nível ou contextuais para indicar o status sócio-econômico, por exemplo: percentual de analfabetos, número de favelas, de estabelecimentos bancários do bairro onde mora cada indivíduo incluído no estudo, etc., o que significa que todos os que moram no mesmo bairro teriam o mesmo valor para esta variável, que seria assim uma agregada. É importante lembrar que nestes modelos, onde variáveis de exposição são consideradas em diversos estágios, os desfechos analisados são sempre considerados no nível primário ou do indivíduo.

Os modelos de regressão tradicionais partem do princípio de que as pessoas estudadas são independentes entre si em relação ao desfecho; todas as variáveis são tratadas como sendo do mesmo estágio hierárquico. Ignorar o papel delas no nível macro pode levar a uma compreensão incompleta dos determinantes das doença nos indivíduos e nas populações (Diez-Roux, 1998b; Victora et al., 1997). As variáveis de grupo ou macro afetam os indivíduos diretamente ou forçam as escolhas feitas por cada um; muitas variáveis medidas indivi- 
dualmente são fortemente condicionadas por processos sociais operando nos níveis de grupos sociais ou sociedades. Quando os dados são estruturados em hierarquias, unidades no mesmo grupo raramente são independentes porque compartilham de um mesmo ambiente e apresentam características semelhantes.

Susser (1994) denominou de mistos os estudos que investigavam efeitos de variáveis independentes ecológicas com as dependentes de nível individual. A incorporação de variáveis de nível de grupo nos modelos de análise, permite que sejam extraídas informações impossíveis de serem obtidas apenas no estágio individual (Diez-Roux, 1998b): por exemplo, a probabilidade de se adotar certo comportamento depende em parte, do grau que este comportamento já foi adotado na comunidade. A análise multinível, junto com outras estratégias e desenhos de estudo, pode contribuir para fortalecer e revitalizar a investigação dos determinantes sociais e coletivos da saúde. Uma boa ilustração disto é o estudo que investigou, por meio de modelos multinível, os efeitos do contexto de vizinhança na prevalência de fatores de risco para doença cardíaca coronariana, em quatro comunidades dos Estados Unidos (DiezRoux et al., 1997). Os resultados mostraram que a variável escolhida para o nível de grupo (bairros) explicaram as associações encontradas: pessoas que moravam em locais precários tiveram risco aumentado de doença cardíaca coronariana, de risco, de hipertensão arterial e de hipercolesterolemia, após ajustamento por indicadores individuais de nível sócio-econômico.

Nos últimos anos percebe-se uma forte tendência de incorporar, dentro de novas linhas de pesquisas ou mesmo em outras em andamento, modelos que consideram os diversos níveis na rede de causalidade (por exemplo: molecular, individual e social), levando a um paradigma que reforça o pensamento sobre causas em níveis múltiplos de organização, dentro de um contexto histórico tanto de sociedades como de indivíduos (Schwartz et al., 1999). Neste sentido os modelos multinível exerceriam um papel que extrapola a técnica estatística de análise, mas que pressupõe uma concepção ou mesmo uma tendência de trabalhar de forma mais dinâmica com o conceito de causalidade. Neste sentido os modelos multinível estariam de fato representando a proposta da eco-epidemiologia multinível proposta por Susser (1998), que teria o potencial de juntar as epidemiologias molecular e global baseado nos sistemas de informação.

\section{Conclusões}

O grande crescimento do conhecimento tecnológico e metodológico dos últimos tempos tem reorientando as práticas de investigação epidemiológica, sobretudo no campo das doenças crônicas. Para alguns tipos de câncer, muito do que se conhece hoje a respeito de fatores de risco (dieta e câncer de cólon, vírus da hepatite B e hepatoma, papilomavírus humano e câncer de colo uterino) foi levantado a partir de comparações internacionais de incidência, entre os anos 50 e 60, e nem sempre as hipóteses eram coerentes com o conhecimento biológico existente na época. Em alguns tipos de câncer ocupacional, como aqueles associados ao benzeno e arsênico, houve uma grande lacuna de tempo para que os achados epidemiológicos fossem confirmados em laboratório (Pearce, 1999). No caso de fumo e câncer de pulmão, por mais de quarenta anos não foi possível estabelecer analogia direta entre os experimentos com animais e os importantes resultados dos estudos epidemiológicos (Susser \& Susser, 1998).

O que acontece atualmente, no entanto, é que os epidemiologistas estão diante de situações diferentes destas. Devido a rapidez com que vem se desenvolvendo a biologia molecular, a elucidação dos mecanismos causais das doenças crônicas que dependem da identificação e quantificação das interações entre os fatores genéticos e exposições ambientais, tende a ser cada vez mais complexa levando a interpretações imprecisas e nem sempre satisfatórias. Será necessário um esforço cada vez maior de integração dos profissionais das diversas áreas (clínica, biologia, epidemiologia e ciências sociais) para que os achados no nível molecular sejam validados por estudos dentro do contexto populacional. Neste sentido, contribuições com diferentes concepções (estudos centrados no nível individual, molecular associados a outros que incorporem variáveis contextuais relacionadas a fatores que afetam grupos de indivíduos ou populações), bem como a utilização de diversas metodologias, que podem englobar tanto análises qualitativas quanto modelos matemáticos mais complexos, podem ser, a princípio, relevantes desde que trabalhados no sentido de se constituírem como elementos a serem recambiados em novas hipóteses e explicações.

\section{Agradecimentos}

À Rosely Sichieri, Sergio Koifman e Victor Wünsch Filho por me incentivarem a publicar estas reflexões. À Dina Czeresnia pelas valiosas críticas e sugestões. 


\section{Referências}

ADAMI, H. O. \& TRICHOPOULOS, D., 1999. Epidemiology medicine and public health. International Journal of Epidemiology, 28:S1005-S1008.

BARKER, D. J., 1990. The fetal and infant origins of adult disease. $B M J, 301: 1111$.

BARKER, D. J., 1995. Fetal origins of coronary heart disease. $B M J, 311: 171-174$.

BARKER, D. J.; BULL, A. R.; OSMOND, C. \& SIMMONDS, S. J., 1990. Fetal and placental size and risk of hypertension in adult life. BMJ, 301:259262.

BARKER, D. J.; MEADE, T. W.; FALL, C. H.; LEE, A.; OSMOND, C.; PHIPPS, K. \& STIRLING, Y., 1992. Relation to fetal and infant growth to plasma fibrinogen and factor VII concentration in adult life. BMJ, 304:148-152.

BARKER, D. J.; OSMOND, C.; GOLDING, J.; KUH, D. \& WADSWORTH, M. E. J., 1989. Growth in utero, blood pressure in childhood and adult life, and mortality from cardiovascular disease. $B M J, 298$ : 564-567.

BARRETO, M. L.; 1998. Por uma epidemiologia da saúde coletiva. Revista Brasileira de Epidemiologia, 1:104-122.

BARROS, F. C. \& VICTORA, C. G., 1999. Increased blood pressure in adolescents who were small for gestational age at birth: A cohort study in Brazil. International Journal Epidemiology, 28:676-681.

DIEZ-ROUX, A. V., 1998a. On genes, individuals, society and epidemiology. American Journal of Epidemiology, 148:1027-1032.

DIEZ-ROUX, A. V., 1998b. Bringing context back into epidemiology: Variables and fallacies in multilevel analysis. American Journal of Public Health, 88:216-22.

DIEZ-ROUX, A. V.; NIETO, F. J.; MUNTANER, C.; TYROLER, H. A.; COMSTOCK, G. W.; SHAHAR, E.; COOPER, L. S.; WATSON, R. L. \& SZKLO, M., 1997. Neighborhood environments and coronary heart disease: A multilevel analysis. American Journal of Epidemiology, 146:48-63.

ELLSWORTH, D.; HALLMAN, M. \& BOERWINKLE, E., 1997. Impact of the human genome project on epidemiologic research. Epidemiologic Reviews, 19:9-11.

HOX, J. J., 1995. Applied Multilevel Analysis. Amsterdam: TT-Publikaties.

HULKA, B.; WILCOSKY, T. C. \& GRIFFITH, J. D., 1990. Biological Markers in Epidemiology. Oxford: University Press.

JOSEPH, K. S. \& KRAMER, M. S., 1996. Review of the evidence on fetal and early childhood antecedents of adult chronic disease. Epidemiologic Reviews, 18:158-174.

KENDLER, K. S., 1997. Social support: A genetic-epidemiologic analysis. American Journal of Psychiatry, 154:1398-1404.

KUH, D. \& BEN-SHLOMO, Y., 1997. A Life Course Approach to Chronic Disease Epidemiology. Oxford: Oxford University Press.

LEON, D. A.; LITHELL, H. O.; VAGERÖ, D.; KOUPILOVÁ, I.; MOHSEN, R.; BERGLUND, L.; LITHELL, U.-B. \& McKEIGUE,. P. M., 1998. Reduced fetal growth rate and increased risk of death from ischaemic heart disease: Cohort study of 15000 Swedish men and women born 1915-29. BMJ, 317:241-245.

PEARCE, N., 1996. Traditional epidemiology, modern epidemiology and public health. Epidemiologia $e$ Prevenzione, 21:92-99.

PEARCE, N., 1999. Epidemiology as a population science. International Journal of Epidemiology, 28: S1015-S1018.

PERUSSE, L.; TREMBLAY, A.; LEBLANC, C. \& BOUCHARD, C., 1989. Genetic and environmental influences on level of habitual physical activity and exercise participation. American Journal of Epidemiology, 129:1012-1022.

PETO, R., 1999. Richard Doll's contribution to Epidemiology. European Journal of Cancer, 35:11.

ROBSON, R. J., 1992. Is the child father of the man? Controversy about the early origins of cardiovascular disease. BMJ, 304:789-790.

SAVITZ, D. A., 1994. In defense of black box epidemiology. Epidemiology, 5:550-552.

SCHWARTZ, S.; SUSSER, E. \& SUSSER, M., 1999. A future for epidemiology? Annual Review of Public Health, 20:15-33.

SHY, C. M., 1997. The failure of academic epidemiology: Witness for the prosecution. American Journal of Epidemiology, 145:479-484.

SUSSER, M., 1994. The logic in ecological: II. The logic of design. American Journal of Public Health, 84:830-835.

SUSSER, M., 1995. The tribulations of trials - Interventions in communities. American Journal of Public Health, 85:156-158.

SUSSER, M., 1998. Does risk factor epidemiology put epidemiology at risk? Peering into the future. Journal of Epidemiology Community Health, 52: 612-613.

SUSSER, M., 1999. Should epidemiologist be a social scientist or a molecular biologist? International Journal of Epidemiology, 28:S1019-1022.

SUSSER, M. \& SUSSER, E., 1996a. Choosing a future for epidemiology: I. Eras and paradigms. American Journal of Public Health, 86:668-673.

SUSSER, M. \& SUSSER, E., 1996b. Choosing a future for epidemiology: II. From black box to Chinese boxes and eco-epidemiology. American Journal of Public Health, 86:674-677.

SUSSER, M. \& SUSSER, E., 1998. Um futuro para a epidemiologia. In: Teoria Epidemiológica HojeFundamentos, Interfaces e Tendências (N. Almeida Filho, M. L. Barreto, R. P. Veras, \& R. B. Barata, org.). pp. 187-212, Rio de Janeiro: ABRASCO.

TRICHOPOULOS, D., 1996. The future of epidemiology. $B M J, 313: 436-437$.

VICTORA, C. G.; HUTTLY, S. R.; FUCHS, S. \& OLINTO, M. T., 1997. The role of conceptual frameworks in epidemiological analysis: A hierarchical approach. International Journal of Epidemiology, 26:224-227 